\title{
Effective Green Semiconductor Lasers with Multiple CdSe/ZnSe QD Active Region for Electron Beam Pumping
}

\author{
S.V. Gronin ${ }^{a}$, M.M. Zverev ${ }^{b}$, S.V. Sorokin ${ }^{a}$, I.V. Sedova ${ }^{a}$, \\ N.A. Gamov ${ }^{b}$, D.V. Peregoudov ${ }^{b}$, V.B. Studionov ${ }^{b}$, \\ P.S. KOP'EV ${ }^{a}$ AND S.V. IVANOV ${ }^{a}$ \\ ${ }^{a}$ Ioffe Physico-Technical Institute of RAS \\ Polytekhnicheskaya 26, St. Petersburg 194021, Russia \\ ${ }^{b}$ Moscow Institute for Radio Engineering, Electronics and Automation \\ Vernadskogo 78, Moscow 119454, Russia
}

The characteristics of ZnSe-based electron beam pumped semiconductor lasers are presented in detail. The laser structures consist of a $0.6 \mu \mathrm{m}$ thick superlattice waveguide centered with ten equidistantly placed CdSe/ZnSe quantum dot active layers. The maximum light output pulse power of $12 \mathrm{~W}$ per facet at room temperature along with an extremely high quantum efficiency of $\approx 8.5 \%$ were obtained at an electron beam pumping energy of $23 \mathrm{keV}$ (the laser wavelength is of $542 \mathrm{~nm}$ ). The calculations of a spatial distribution of non-equilibrium carrier concentration within the semiconductor structures under electron beam pumping are presented. The possible ways of further improvement of laser efficiency are discussed.

PACS numbers: 78.55.Et, 78.67.Hc

\section{Introduction}

Development of semiconductor lasers emitting in green part of the visible spectrum are still of great interest due to a wide area of potential applications such as medical equipment, navigation and positioning systems, local networks etc. However, the commercial manufacturing of semiconductor green laser diodes is currently impracticable due to a set of unsolved problems of fabrication and operation of such lasers. Among them are the maintenance of high degradation stability of laser diodes based on wide-gap II-VI compounds at high $p$-type doping of $\mathrm{ZnSe}$ with nitrogen. 
Electron beam pumped (EBP) lasers using ZnSe-based structures as active elements look very promising for the development of green lasers, as no requirements of low-resistivity contacts and $p-n$ junction are applied. Moreover, the maximum conversion efficiency of electron beam energy to light is as high as $30 \%$. The non-equilibrium carriers are generated within the relatively large volume of the material, which, in turn, is governed by the penetration depth of the electron beam into the crystal as well as its cross-section. EBP laser provides the ability to vary the thickness of excited region and output light power within the wide range by adjusting the electron beam energy. The EBP laser also allows the wide-range modulation of light output power, the electronic control of the directivity diagram, and synchronization of light and pump pulses at a level of a fraction of nanosecond. Nevertheless, there are several limitations for single crystal-based EBP lasers such as the high threshold current density and energy of electron beam.

During the last decades several successful attempts in fabrication of quantum-sized ZnSe-based EBP lasers were demonstrated [1]. Using of lowdimensional heterostructures with short-period superlattice (SL) waveguide and single $\mathrm{CdSe} / \mathrm{ZnSe}$ quantum dot $(\mathrm{QD})$ active region resulted in room-temperature lasing at $E_{\mathrm{e}}<5 \mathrm{keV}$ with the extremely low threshold current density $j_{\text {th }}=$ $0.4-0.5 \mathrm{~A} / \mathrm{cm}^{2}\left(E_{\mathrm{e}}=8-10 \mathrm{keV}\right)[2]$. The quantum efficiency of $\approx 4 \%$ as well as light output power of $>10 \mathrm{~W}$ (per facet) have been achieved in a laser structure with extended (0.4 $\mu \mathrm{m}$ thick) SL waveguide and three ZnSe/CdSe QD sheets [3].

This paper presents the recent results on fabrication and study of green EBP ZnSe-based laser heterostructures with 10 active CdSe/ZnSe QD sheets equidistantly placed within an extended ZnSe/ZnSSe SL waveguide. The structure design has been optimized to achieve both the maximum quantum efficiency and output pulse power at a reasonable $E_{\mathrm{e}}$. The values of pulse output power of $12 \mathrm{~W}$ and the quantum efficiency of $8.5 \%$ per facet have been demonstrated.

\section{Experiment}

$\mathrm{ZnMgSSe} / \mathrm{ZnSSe} / \mathrm{Zn}(\mathrm{Cd}) \mathrm{Se}$ laser heterostructures were grown by molecular beam epitaxy (MBE) pseudomorphically to GaAs(001) substrate via a GaAs buffer epilayer at substrate temperature $T_{\mathrm{s}}=270-280^{\circ} \mathrm{C}$. The structure consists of bottom and top $\mathrm{Zn}_{0.9} \mathrm{Mg}_{0.1} \mathrm{~S}_{0.15} \mathrm{Se}_{0.85}$ cladding layers with the thickness of $1.65 \mu \mathrm{m}$ and $20 \mathrm{~nm}$, respectively, and the $2.4 \mathrm{~nm}-\mathrm{ZnS}_{0.15} \mathrm{Se}_{0.85} / 0.9 \mathrm{~nm}-\mathrm{ZnSe} \mathrm{SL}$ waveguide with the whole thickness of $\approx 0.65 \mu \mathrm{m}$. The bottom cladding was intentionally $n$-type doped with $\mathrm{Cl}$. The $10 \mathrm{CdSe} / \mathrm{ZnSe} \mathrm{QD}$ sheets were equidistantly placed within the SL waveguide, the nominal thickness of CdSe layers is as high as 2.5 ML [4].

All measurements were carried out at $300 \mathrm{~K}$ in a transverse excitation geometry. No reflecting coatings on the facets were used. The parameters of electron beam were the following: energy $E_{\mathrm{e}}=10 \div 30 \mathrm{keV}$, pulse duration time of $\approx 50 \mathrm{~ns}$, repetition rate up to $10 \mathrm{~Hz}$. The $0.25 \mathrm{~mm}$ size diaphragm has been used to limit 
the electron beam size in the direction normal to laser cavity axis. The lasing spectra were measured using CCD-rules-based recorder mounted at the output of MDR-2 monochromator. The calibrated coaxial photocell has been used to measure both the shape of light pulse and light output power.

\section{Results and discussion}

Figure 1 presents the schematic energy diagram of the laser structure along with the spatial distribution of an electron beam losses within ZnSe at different electron beam energies $E_{\mathrm{e}}(7,15,24$, and $30 \mathrm{keV})$. The lasing has been observed at the wavelength of $542 \mathrm{~nm}$, with the FWHM of the emission line being of $\approx 2-3 \mathrm{~nm}$. The spectrum of laser generation at $E_{\mathrm{e}}=13 \mathrm{keV}$ is shown in the inset.

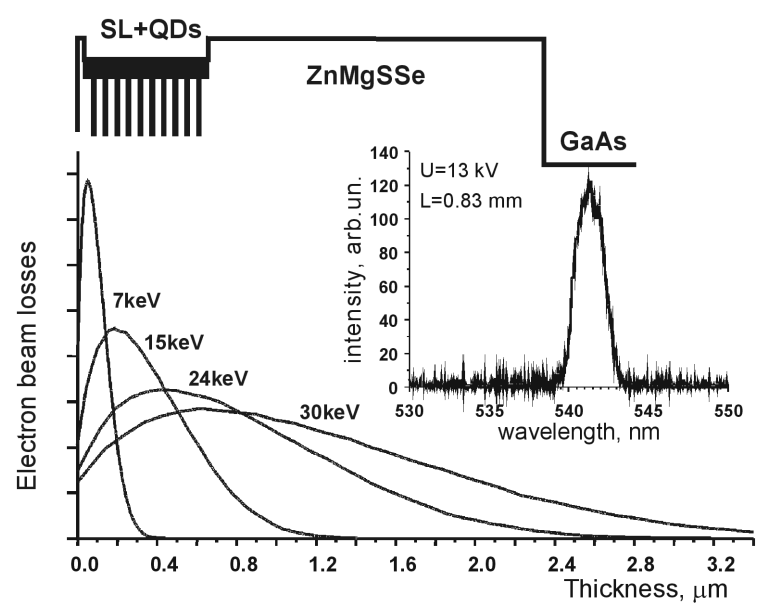

Fig. 1. Schematic energy diagram of the laser structure along with spatial distribution of electron beam losses within ZnSe at different electron beam energies $(7,15,24$, and $30 \mathrm{keV}$ ). The laser generation spectrum at $E_{\mathrm{e}}=13 \mathrm{keV}$ is shown in the inset.

The dependences of pulsed output power per facet on electron beam current $\left(I_{\mathrm{e}}\right)$ measured at different electron beam energies are shown in Fig. 2. The cavity length and the width of laser crystal are $L=0.46 \mathrm{~mm}$ and $d=0.25 \mathrm{~mm}$, respectively. The maximum output power of $12 \mathrm{~W}$ is achieved at electron beam energy $E_{\mathrm{e}}=23 \mathrm{keV}$ and current $I_{\mathrm{e}}=5.5 \mathrm{~mA}$. It should be noted that the total current of electron gun in our experiments (because of electron gun design) decreases with a decrease in accelerating voltage due to the effect of e-beam space charge on $I_{\mathrm{e}}$. Therefore at high accelerating voltages there is a possibility to exceed significantly the laser threshold and, consequently, enhance the laser output power. However, using of high $E_{\mathrm{e}}$ may result in an increase in carrier losses within the substrate.

Figure 3 demonstrate the dependence of emission efficiency on electron beam current at electron beam energy of 23 and $26 \mathrm{keV}$ and on electron beam energy at a constant current $(5.7 \mathrm{~mA})$. The maximum efficiency $(\approx 8.5 \%)$ per facet has been 


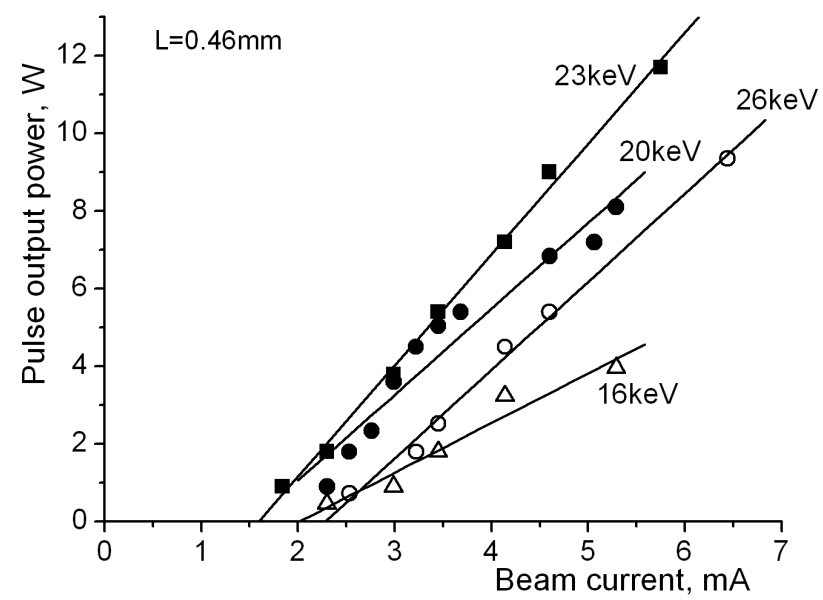

Fig. 2. Dependences of pulse output power on electron beam current registered at different electron beam energies.

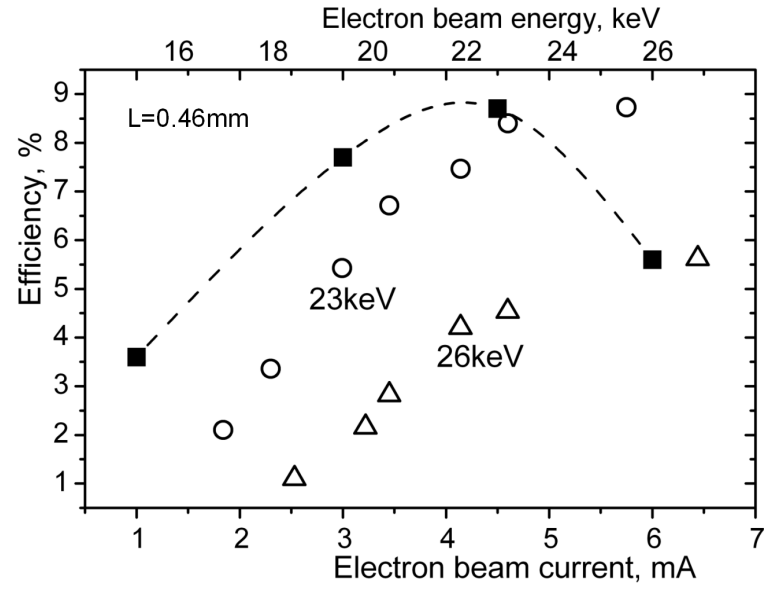

Fig. 3. Dependences of emission efficiency on electron beam current at electron beam energies of 23 and $26 \mathrm{keV}$. The filled squares reflect the maximum emission efficiency versus electron beam energy.

obtained at electron beam energy and current of $23 \mathrm{keV}$ and $5.7 \mathrm{~mA}$, respectively [6]. This value is extremely high for the EBP lasers. To obtain the real value of the efficiency we should double the recorded value taking into account two laser facets. The main reason of the increase in laser efficiency is likely the more effective utilizing of pumping energy owing to the adjustment of the structure thickness and penetration depth of electron beam into the sample.

The plots presented in Fig. 2 demonstrate no saturation of light output power with rising the electron beam current, though the $I_{\mathrm{e}}$ value was raised to 
its maximum at any $E_{\mathrm{e}}$ for a given e-gun design. Presumably the increase in $I_{\mathrm{e}}$ keeping constant $E_{\mathrm{e}}$ within the $20-30 \mathrm{keV}$ range should allow the further increase in light output power. The optimum number of active CdSe QD sheets to reach maximum light output power and efficiency is also under consideration. Anyway, it is governed by both the electron beam energy matched to the waveguide thickness and the carrier diffusion length, since the latter should be less than the distance between the neighboring QD sheets.

The results of calculations of carrier distribution within laser heterostructure at different electron beam pumping energies are summarized in Fig. 4. Diffusion of carriers as well as their spatial redistribution within the structure due to different band gap energies in different layers have been taken into account. The value of carrier diffusion length was assumed to be equal to $0.5 \mu \mathrm{m}$ [7]; carrier lifetimes have been taken as 0.04 and 0.4 ns in wide-gap ZnMgSSe [8] and waveguide layers, respectively. One should note that the values provide reasonable agreement between calculated and experimental dependence of the cathodoluminescence intensity on electron beam energy [8]. A spatial distribution of electron beam energy losses within ZnSe has been taken from Ref. [5].

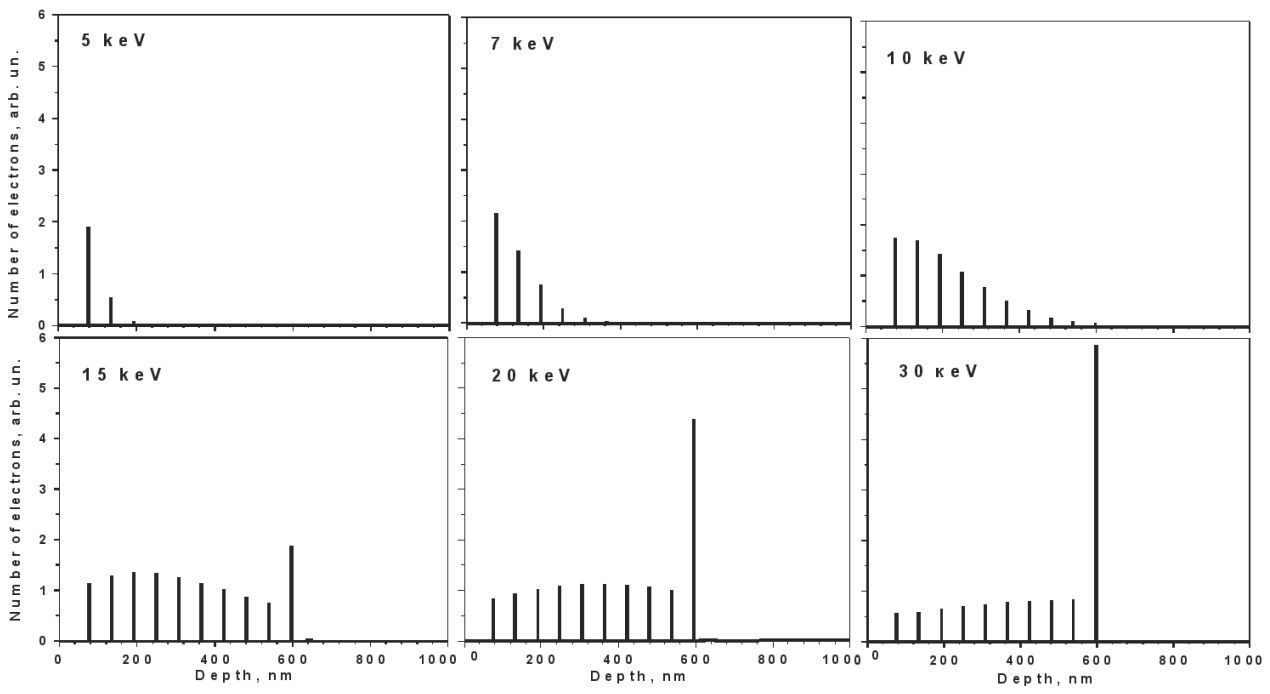

Fig. 4. Calculations of carrier spatial distribution in the structure with 10 active CdSe/ZnSe QD sheets at different pumping electron beam energies. Structure surface is on the left side, inner waveguide surface is located at the $0.65 \mu \mathrm{m}$ depth.

The number of active layers filled with carriers increases with the increase in electron beam energy. It is clearly seen that CdSe QD layers are filled with carriers as the e-beam energy exceeds $15 \mathrm{keV}$. Further increase in e-beam energy will cause the appreciable part of the energy to be absorbed in the wide-gap ZnMgSSe layer, that results in high carrier concentration in the 10th QD layer 
due to efficient carrier diffusion from the cladding layer. At the same time, a part of carriers still remains in the SL layers. However, their concentrations are 2-3 orders of magnitude lower than that in CdSe/ZnSe QD layers. This indicates the efficient transport of carriers in the SL waveguide and their capture into the active layers of laser structure. The carrier distribution at $E_{\mathrm{e}}=15 \mathrm{keV}$ is presented in

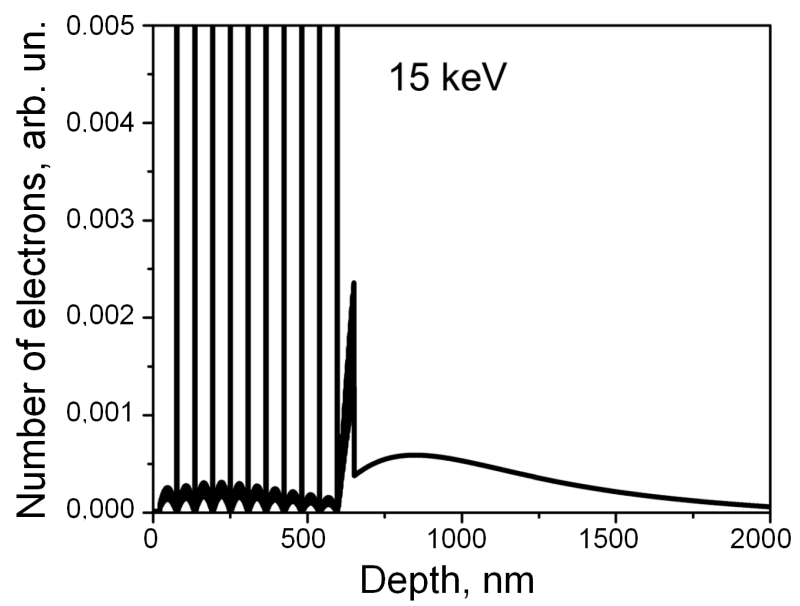

Fig. 5. Carrier distribution within the structure at the electron energy equal to $15 \mathrm{keV}$ with 3 times lower and twice greater scale for $y$ and $x$ axis, respectively.

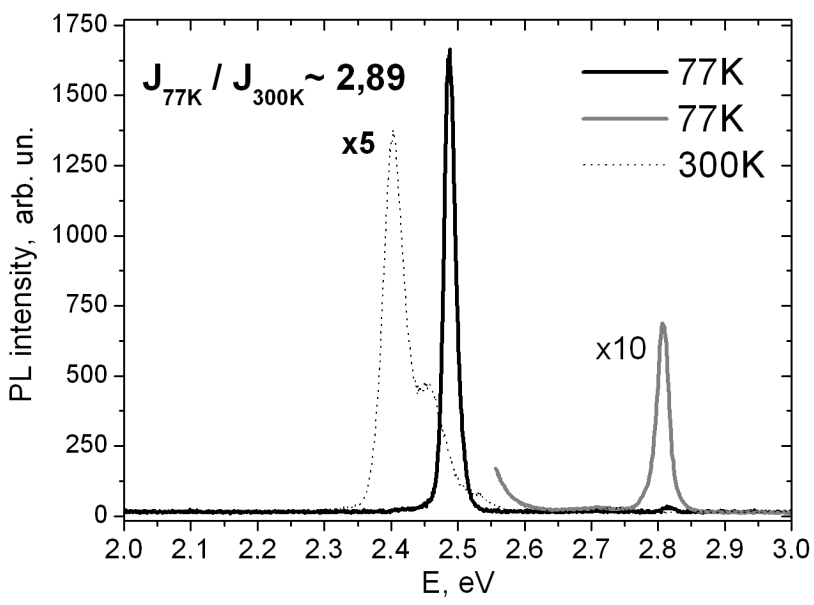

Fig. 6. PL spectrum of optimized structure measured at $77 \mathrm{~K}$ and room temperature. Gray line represents a peak from the SL waveguide.

Fig. 5 at higher magnification. It is clearly seen that negligible part of carriers is concentrated within the SL and lower ZnMgSSe cladding layers. With the increase in electron beam energy the portion of the energy absorbed in the bottom 
cladding layer and, in turn, the carrier concentration in this layer increase, while the efficiency of laser generation decreases. One should note that the true values of carrier concentration in each layer depend on the carrier lifetime, diffusion length, rate of nonradiative recombination at every interface, etc. Nevertheless, the results of calculations allow one to qualitatively estimate the processes in EBP laser structure with multiple QD sheets.

For further optimization a structure with increased distance between QD sheets (up to $0.1 \mu \mathrm{m}$ ) and, consequently, extended waveguide was grown. Figure 6 presents photoluminescence (PL) spectra of the optimized structure at $77 \mathrm{~K}$ and $300 \mathrm{~K}$. The PL intensity of the structure was increased in comparison with the previous one mentioned above. It is clearly seen that integral PL intensity at the room temperature is only about 3 times lower than cryogenic one.

\section{Conclusions}

To summarize, the parameters of EBP ZnSe-based lasers with SL waveguide and $10 \mathrm{CdSe} / \mathrm{ZnSe} \mathrm{QD}$ sheets in the active region have been studied in detail. Extremely high value of quantum efficiency up to $8.5 \%$ and output pulsed power up to $12 \mathrm{~W}$ per facet have been obtained at electron beam energy and current of $23 \mathrm{keV}$ and $I_{\mathrm{e}}=5.7 \mathrm{~mA}$, respectively. The room temperature lasing has been demonstrated at a wavelength of $542 \mathrm{~nm}$. The registered value of quantum efficiency $(\approx 17 \%)$ is close to its theoretical limit $\approx 30 \%$. The main reason of the efficiency increase is provided obviously by an improvement in utilizing the pumping energy in the structure. The possible ways of the laser structure optimization in accordance with calculations of carrier distribution are given.

\section{Acknowledgments}

The work was partly supported by RFBR grants and FASI Program.

\section{References}

[1] D. Herve, R. Accomo, E. Molva, L. Vanzetti, J.J. Paggel, L. Sorba, A. Francioci, Appl. Phys. Lett. 67, 9, 2144 (1995).

[2] M.M. Zverev, N.A. Gamov, E.V. Zhdanova, D.V. Peregudov, V.B. Studenov, S.V. Ivanov, I.V. Sedova, S.V. Sorokin, S.V. Gronin, P.S. Kop'ev, Tech. Phys. Lett. 33, 1032 (2007).

[3] M.M. Zverev, S.V. Sorokin, I.V. Sedova, S.V. Ivanov, D.V. Peregudov, P.S. Kop'ev, Phys. Status Solidi C 2, 923 (2005).

[4] S.V. Ivanov, P.S. Kop'ev, A.A. Toropov, Usp. Fiz. Nauk 169, 468 (2099) (in Russian).

[5] E.N. Donskoy, E.V. Zhdanova, A.N. Zalyalov, M.M. Znerev, S.V. Ivanov, D.V. Peregudov, O.N. Petrushin, Y.A. Savelev, I.V. Sedova, S.V. Sorokin, M.D. Tarasov, Y.N. Shigaev, J. Quantum Electron., in press. 
[6] M.M. Zverev, N.A. Gamov, E.V. Zhdanova, D.V. Peregudov, V.B. Studenov, I.V. Sedova, S.V. Gronin, S.V. Sorokin, S.V. Ivanov, P.S. Kop'ev, Semicond. Lett., in press.

[7] V.I. Kozlovsky, Yu.G. Sadofyev, J. Vac. Sci. Technol. B 18, 1538 (2000).

[8] M.M. Zverev, D.V. Peregoudov, E.V. Zdanova, N.A. Gamov, V.B. Studionov, S.V. Ivanov, S.V. Sorokin, I.V. Sedova, P.S. Kop'ev, Le Si Dang, in: Proc. 14th Int. Symp. "Nanostructures: Physics and Technology", Ioffe Physical-Technical Institute, St. Petersburg 2006, p. 27. 